\title{
A Case of Recurrent Fixed Drug Eruption Secondary to Desloratadine
}

\author{
Eman Almukhadeb ${ }^{1}$ \\ 1. Dermatology, College of Medicine, King Saud University, Riyadh, SAU
}

Corresponding author: Eman Almukhadeb, eman-derma@hotmail.com

\begin{abstract}
Desloratadine is a second-generation H1-type antihistamine that is widely prescribed for multiple indications including allergic rhinitis and urticaria. It is well-tolerated and is not known to cause cutaneous side effects including fixed drug eruption (FDE). In this report, a case of recurrent fixed drug eruption induced by desloratadine is reported.
\end{abstract}

Categories: Dermatology

Keywords: desloratadine, anti-histamine, fixed drug eruption, cutaneous drug eruption, delayed hypersensitivity

\section{Introduction}

Fixed drug eruption (FDE) is a type of cutaneous drug eruption that is characterized by single or multiple tenders or pruritic, well-demarcated, round-oval erythematous edematous plaques. It may develop a dusky violaceous hue or central bullae that later end up with erosion secondary to epidermal detachment [1]. It tends to develop few days to two weeks after the initial exposure to certain medication. It appears at the same site within 24 hours upon re-administration of the causative drug. Lesions can appear anywhere on the skin, as well as the mucous membranes, with common sites being the lips, face, hands, feet, and genitalia. When the lesions fade away, they often leave a residual post-inflammatory hyperpigmentation. Recurrence in the same location upon re-administration of a particular medication is the key to diagnosis. Provocation via patch testing in a previously involved site might be useful in determining the responsible drug [1,2].

Review began 07/17/2021 Review ended 07/20/2021 Published 07/30/2021

(c) Copyright 2021

Almukhadeb. This is an open access article distributed under the terms of the Creative Commons Attribution License CC-BY 4.0., which permits unrestricted use, distribution, and reproduction in any medium, provided the original author and source are credited.
The exact mechanism of FDE is unknown. Recent research suggests a cell-mediated process that initiates both the active and quiescent lesions. The process may involve an antibody-dependent, cell-mediated cytotoxic response. CD8+ effector/memory T cells play an important role in the reactivation of lesions with re-exposure to the offending drug $[3,4]$.

The drugs most commonly associated with FDE include sulfonamides, tetracyclines, $\beta$-lactams, fluoroquinolones, macrolides, non-steroidal anti-inflammatory drugs (NSAIDs), acetaminophen, aspirin, barbiturates, dapsone, proton pump inhibitors, and azole antifungal drugs [5]. There are few reports of antihistamines as a cause of FDE [6-12].

Desloratadine is a selective $\mathrm{H} 1$ receptor antagonist and is the primary metabolite of loratadine. The literature review showed no reported cases of FDE secondary to desloratadine.

\section{Case Presentation}

A 59-year-old female was presented to the dermatology clinic at King Khaled University Hospital, Riyadh, with a one-year history of few slightly pruritic skin eruptions over arms and thighs. She is known to have diabetes mellitus controlled on metformin for the last nine years, hypertension controlled on nifedipine and irbesartan for the last seven years, and allergic rhinitis on mometasone furoate nasal inhaler and on-off desloratadine. She started to take desloratadine in February 2020 and she only takes it when her rhinitis symptoms flare up. The patient noticed that whenever she takes desloratadine she starts to develop these lesions at the same locations and they clear up in few days leaving post-inflammatory hyperpigmentation. On examination, she has five, well-defined, round, variable in size, erythematous to violaceous patches and plaques over the left thigh and both arms as shown in Figure 1. Mucous membranes were free. 


\section{Cureus}
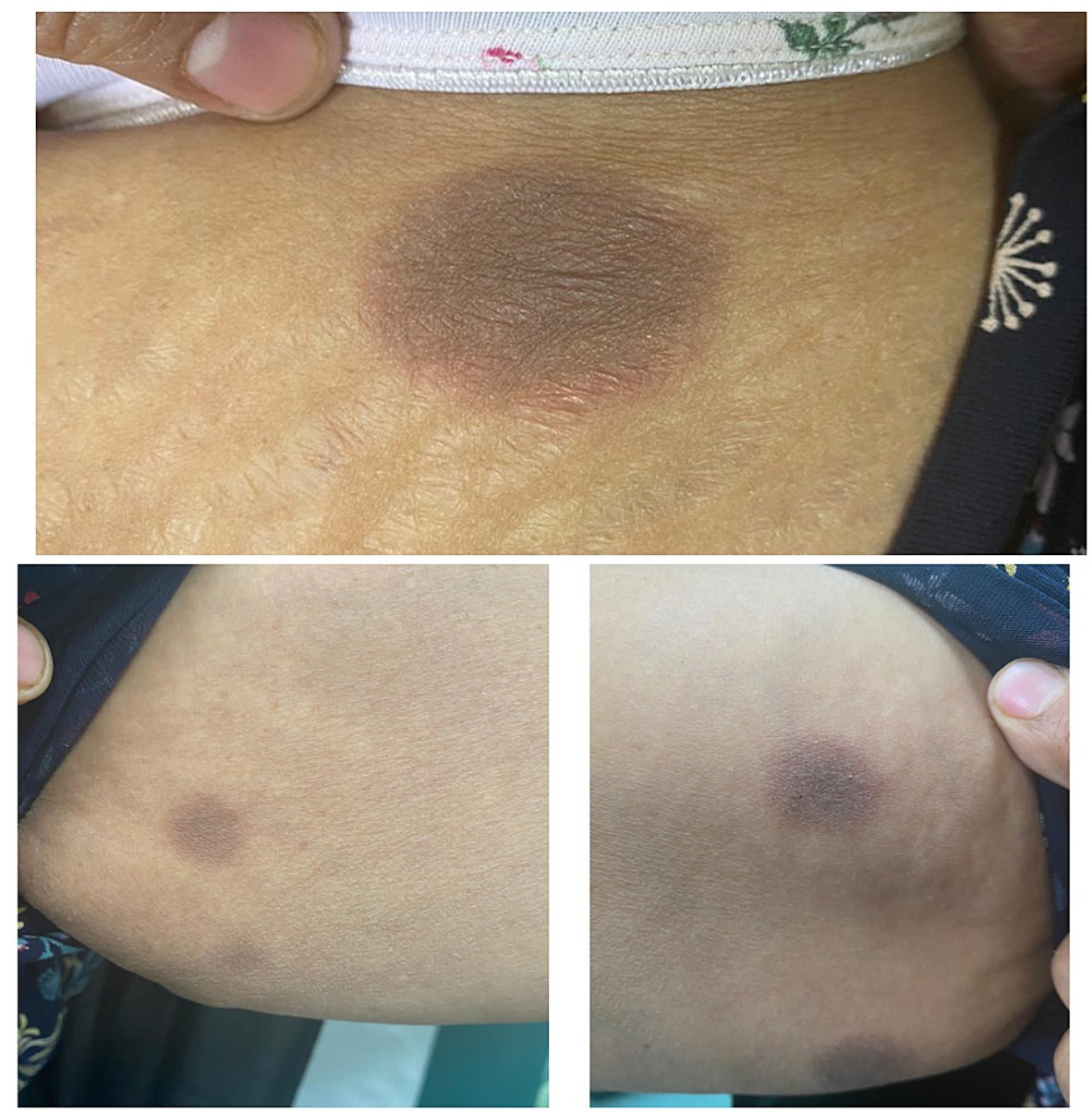

FIGURE 1: Well-defined, erythematous to violaceous patches and plaques over left arm, left thigh, and right arm.

A skin biopsy was obtained from the lesion at the right arm. Histologic examination revealed a normal basket weave stratum corneum, interface dermatitis, scattered necrotic keratinocyte, perivascular lymphocytes, dermal eosinophils, and melanophages consistent with the diagnosis of FDE, as shown in Figure 2. 


\section{Cureus}

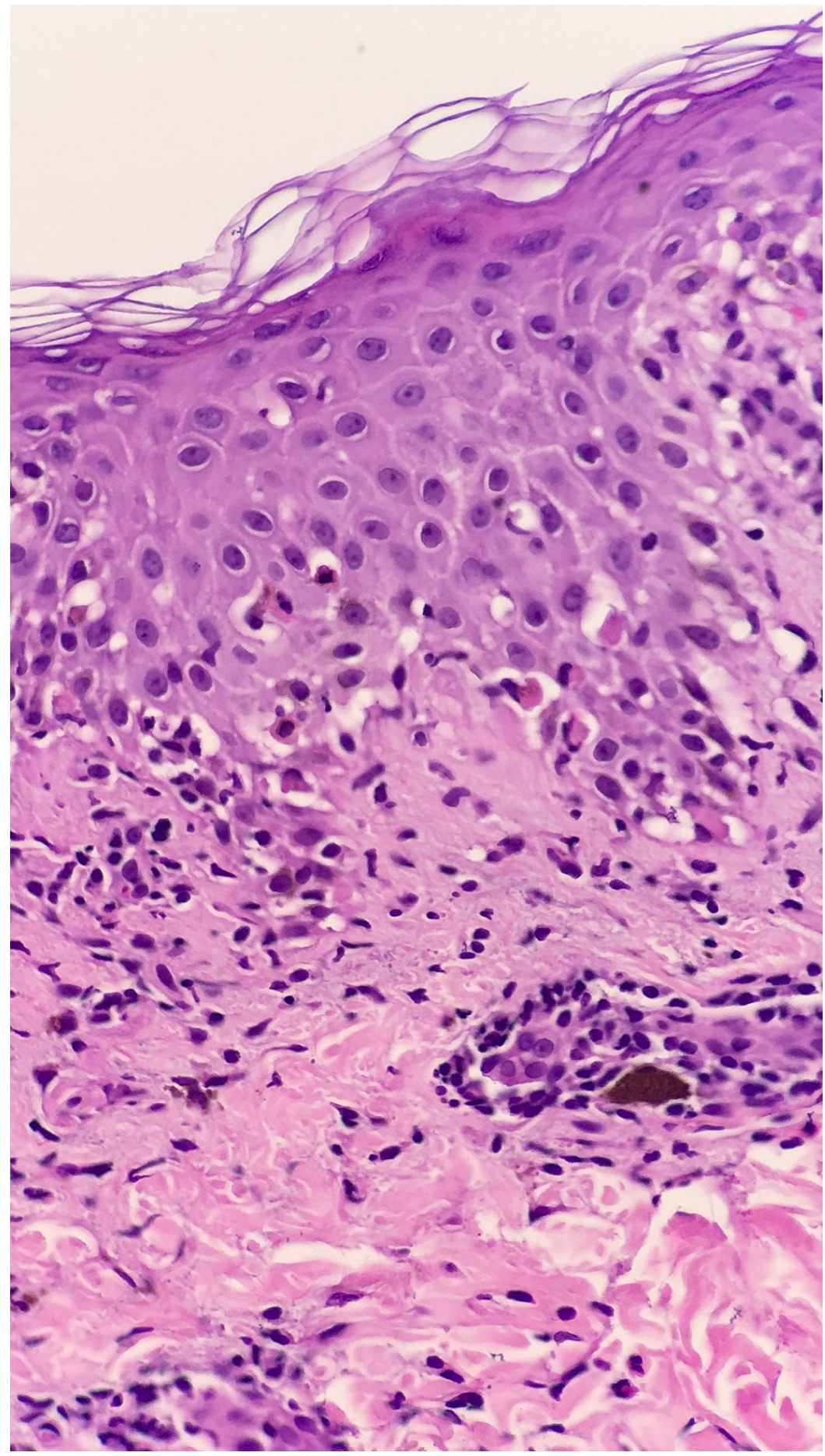

FIGURE 2: Interface dermatitis, scattered necrotic keratinocyte, dermal eosinophils, and melanophages.

The patient was referred to an allergy clinic for patch testing of the involved site which was provoked by the application of desloratadine. The patient was given topical mometasone furoate $0.1 \%$ ointment and advised to stop desloratadine. Two months later, her skin examination showed only post-inflammatory hyperpigmentation as shown in Figure 3, for which she was given hydroquinone $4 \%$ cream. 


\section{Cureus}

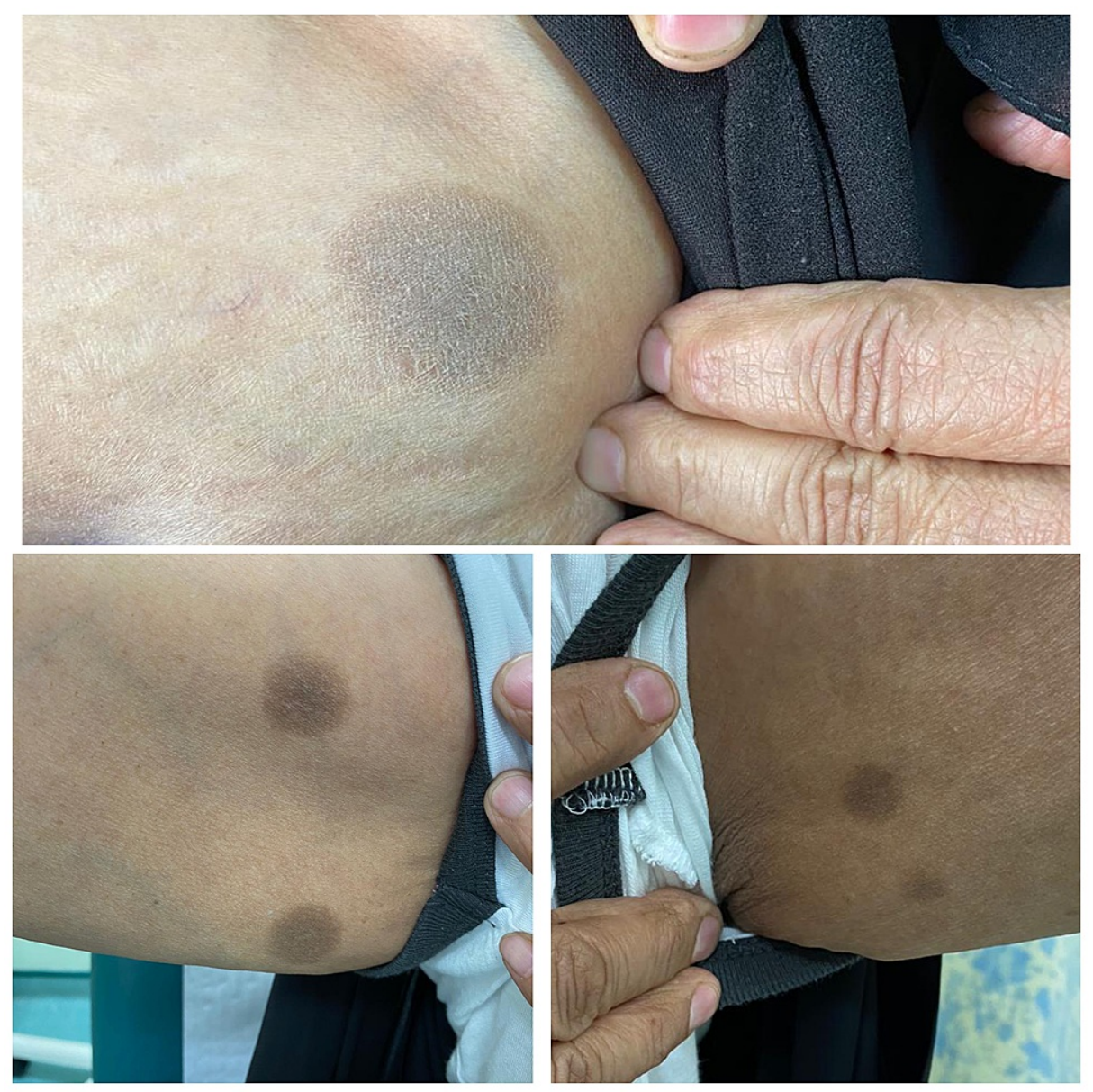

\section{FIGURE 3: Two months after discontinuation of desloratadine with only post-inflammatory hyperpigmentation over left arm, right arm, left thigh.}

\section{Discussion}

Fixed drug eruption is an uncommon type of cutaneous adverse drug eruption. It can be triggered by a large number of drugs including antibiotics, antiepileptics, nonsteroidal anti-inflammatory agents, phenothiazines, and other agents, and also certain foods such as cashews and licorice have also been reported as causative agents [5].

H1-antihistamines are widely used in clinical practice for the treatment of allergic disorders such as allergic rhinitis, urticaria, and other pruritic disorders. They have an excellent safety profile and are uncommonly associated with adverse cutaneous drug eruptions. FDE induced by first and second-generation H1antihistamine is rare. There are few reports of FDE caused by hydroxyzine, cetirizine, diphenhydramine, dimenhydrinate, and loratadine [6-12].

A Medline search revealed no reported cases of FDE secondary to desloratadine and only two cases of FDE were induced by loratadine $[8,9]$. Since desloratadine is the active metabolite of loratadine, this study supports the previous two case reports of loratadine-induced FDE. Furthermore, it is expected that patients who developed FDE to desloratadine develop an FDE to loratadine as the first is the active metabolites of the second and it is recommended to use another antihistamine in such a patient.

In this case report, the diagnosis of FDE due to desloratadine was established based on recurrent episodes related to intermittent administration of desloratadine, i.e., positive oral rechallenge which is the gold standard for diagnosis, as well as a patch test, clinical and histologic findings.

The exact mechanism by which desloratadine induces FDE is unknown. It is thought that desloratadine act as a hapten that binds to basal keratinocytes, leading to an inflammatory response. This leads to the secretion of cytokines from keratinocytes including intercellular adhesion molecule-1 (ICAM1). ICAM1 helps $\mathrm{T}$ cells (CD4 and CD8) migrate to the insult site. CD8-T cells become reactivated after re-exposure to desloratadine and produce inflammatory cytokines, interferon-gamma, and tumor necrosis factor-alpha which leads to epidermal damage. 
Oral rechallenge should not be routinely performed in FDE especially when positive patch testing brings additional evidence of the culpability of the drug. Oral rechallenge may involve major discomfort for the patient and may increase the occurrence of new lesions.

\section{Conclusions}

In conclusion, it was assumed that this is the first report of the relationship between the oral ingestion of desloratadine and the production of a typically FDE. Since desloratadine is the active metabolite of loratadine, this patient might develop similar lesions if she takes loratadine in the future. So it is recommended to use different antihistamines other than loratadine and desloratadine.

\section{Additional Information}

\section{Disclosures}

Human subjects: Consent was obtained or waived by all participants in this study. IRB Committee at College of Medicine, King Saud University issued approval N/A. Conflicts of interest: In compliance with the ICMJE uniform disclosure form, all authors declare the following: Payment/services info: All authors have declared that no financial support was received from any organization for the submitted work.

Financial relationships: All authors have declared that they have no financial relationships at present or within the previous three years with any organizations that might have an interest in the submitted work. Other relationships: All authors have declared that there are no other relationships or activities that could appear to have influenced the submitted work.

\section{Acknowledgements}

This research is supported by the College of Medicine Research Center, Deanship of Scientific Research, King Saud University.

\section{References}

1. Lipowicz S, Sekula P, Ingen-Housz-Oro S, et al.: Prognosis of generalized bullous fixed drug eruption: comparison with Stevens-Johnson syndrome and toxic epidermal necrolysis. Br J Dermatol. 2013, 168:72632. 10.1111/bjd.12133

2. Stubb S, Alanko K, Reitamo S: Fixed drug eruptions: 77 cases from 1981 to 1985 . Br J Dermatol. 1989, 120:583. 10.1111/j.1365-2133.1989.tb01337.x

3. Mizukawa Y, Shiohara T: Fixed drug eruption: a prototypic disorder mediated by effector memory $\mathrm{T}$ cells . Curr Allergy Asthma Rep. 2009, 9:71-7. 10.1007/s11882-009-0011-8

4. Shiohara T: Fixed drug eruption: pathogenesis and diagnostic tests . Curr Opin Allergy Clin Immunol. 2009, 9:316-21. 10.1097/ACI.0b013e32832cda4c

5. Sehgal VN, Srivastava G: Fixed drug eruption (FDE): changing scenario of incriminating drugs . Int J Dermatol. 2006, 45:897-908. 10.1111/j.1365-4632.2006.02853.x

6. Fellner MJ: Clinics in Dermatology: Unexpected Drug Reactions . J.B. Lippincott, Philadelphia; 1986.

7. Ackerman AB: Atlas Clínico de las 101 Enfermedades más Comunes de la Piel . Marbán, Madrid; 2002.

8. Ruiz-Genao DP, Hernández Nuñez A, Sánchez Pérez J, Garcia Diez A: Fixed drug eruption due to loratadine. Br J Dermatol. 2002, 146:528-9. 10.1046/j.1365-2133.2002.46314.x

9. Pionetti CH, Kien MC, Alonso A: Fixed drug eruption due to loratadine. Allergol Immunopathol (Madr). 2003, 31:291-3. 10.1016/s0301-0546(03)79199-X

10. Kränke B, Kern T: Multilocalized fixed drug eruption to the antihistamine cetirizine. J Allergy Clin Immunol. 2000, 106:988. 10.1067/mai.2000.110791

11. Dwyer CM, Dick D: Fixed drug eruption caused by diphenhydramine. J Am Acad Dermatol. 1993, 29:496-7. 10.1016/s0190-9622(08)82008-7

12. Cohen HA, Cohen Z, Frydman M: Fixed drug eruption of the scrotum due to hydroxyzine hydrochloride (Atarax). Cutis. 1996, 57:431-2. 